\title{
Stability test of TCRP1201+ total station parameters and its setup
}

\author{
Waldemar Odziemczyk ${ }^{1, *}$ \\ ${ }^{1}$ Warsaw University of Technology, Faculty of Geodesy and Cartography, 1 Politechniki Sq., 00-661 Warszawa, Poland
}

\begin{abstract}
Monitoring systems utilising automated electronic total stations are an important element of controlling safety levels of numerous engineering structures. In order to ensure appropriate credibility of the obtained data, it is crucial to maintain the stability of both the parameters of the used instrument as well as of its setup. This study presents the results of research on the stability of the Leica TCRP1201+ instrument's basic parameters and three selected tripods manufactured using different materials. In the case of the total station the inspected values included Hz-collimation, V-collimation, and tilt compensation errors. Two of the head tilt components and torsion were examined for each of the tripods. The research was carried out in three-day sessions in conditions of large temperature fluctuations. Graphs presenting the changes in the studied values were supplemented with analysis of correlation between the said changes and the changes in temperature. The conclusions section of the study includes suggestions on how to avoid the negative effects of changes in the geometry of the measuring device.
\end{abstract}

\section{Electronic Total stations in deformation monitoring}

Geodetic monitoring of deformations/displacements is an important element of the system ensuring safety of numerous types of engineering structures. It takes advantage of various surveying techniques, such as satellite measurement, photogrammetry, laser scanning, and inclinometric or classic linear and angular measurements. Studies [1-3] contain selected examples of surveying systems utilising the last of the aforementioned techniques. A significant advantage of the measurement systems based on the geodetic linear and angular surveys lies in the combination of a relatively high accuracy, its independence from the availability of the satellite signal and the ability to determine absolute displacements referred to an external, from the perspective of the surveyed object, reference system.

Sufficient accuracy of the utilised total station is the first condition for obtaining valuable data of the object state. Papers [4-6] contain information on various methods of investigating the accuracy of instruments. Stability of the total station setup and the points defining the reference system is the prerequisite for the acquisition of adequately accurate and reliable displacement values for the inspected object. In the case of structures subjected to constant monitoring the station setup is usually located on a massive concrete pillar ensuring adequate stability. The situation is somewhat different when carrying out emergency measurements calling for the ability to start the monitoring immediately. As there is not time (and sometimes place) to construct a concrete pillar, the instrument is placed on a tripod. The stability of a total station setup in such a manner depends on a number of factors (type of the tripod, type of the base, changes in the weather conditions) and the possible changes should be taken into consideration when planning the survey and processing the obtained results.

Notwithstanding the foregoing, one should take into consideration the occurrence of changes in the parameters of the instrument itself. In particular it may concern the change in the orientation of line of sight in regard to the telescope which manifests itself as $\mathrm{Hz}$ - and $\mathrm{V}$-collimation changes and zero point of the electronic vial used for compensation of the influence of tilt of the instrument's vertical axis.

This study is a continuation of research described in [7] and presents the results of an experiment aimed at researching the changes of the basic parameters of the measuring position and the instrument in the conditions of a several-day-long test survey. Leica TCRP1201+ total station and three types of tripods - aluminium, composite and wooden - were used in the experiment.

Changes in the following parameters were analysed within the framework of this research:

1. For the instrument

- Hz- and V-collimation,

- $\quad$ zero point of the electronic vial (tilt compensation system),

2. For each of the tripods

- Instrument's tilt

- Changes in the orientation of the horizontal circle

The test site was selected so that the measurements coincided with largest possible changes in the temperature. 


\section{Measurement tests}

Measurement test were carried out inside the skylight located in the great hall of the Warsaw University of Technology. The skylight's structure consists of a steel frame and two shells - internal and external. The external shell is made of glass whereas the internal one is made of plastic. On sunny days the enclosed space between the shells causes significant temperature fluctuations. In such conditions changes in the instrument's and the measuring position's parameters would be larger and easier to notice. On the other hand, the limited air exchange inside the skylight causes the changes in the relative humidity to be closely correlated to the changes in temperature.

Both the construction of the skylight and the performance of the surveying instrument were the subject of an earlier research, the results of which were described in [7-10].

Automated Leica TCRP1201+ total station was used in order to carry out the measurement tests. The improved, as compared to the previous versions, ATR (automatic target recognition) system of this instrument was described in [11]. Its software is based on Leica TPS1200 operation system and it allows for control over the work of the total station via GSI interface. This solution was used in TC-calc software working on PC (Windows) in order to carry out the measurement tests. Detailed information on the TC-calc software can be found in the author's earlier papers [12] and [13]. The ability to read the instrument's current tilt value turned out to be a particularly important feature of the TCRP1201+ total station.
The stability of the total station's setup also depends on the quality of the tripod. It is particularly important in the case of instruments equipped with servomotors. The effects of dynamic loads occurring at the beginning and the end of rotation of the instrument were the subject of a study by C. Depenthal [14].

During the test the total station was successively placed on three tripods: aluminium (Leica), composite (Nedo) and wooden (Leica). All of them were standard tripods. It was ensured that all the screws were properly tightened and that all clearances were eliminated on all of the interconnecting parts of each of the tripods.

The measurement test entailed automatic measurement of two points 1 and 2 in complete series every $30 \mathrm{~min}$. The instruments setup was located on a concrete pillar supporting the structure of the skylight. The locations of points 1 and 2 were selected in such a way, that the lines of sight to them formed a right angle (Fig. 1). The measurement took 64 to 72 hours, which allowed for the observation of several cycles of daily temperature fluctuations.

Horizontal and vertical angles were registered during the measurements $(\mathrm{Hz}, \mathrm{V})$ and the tilt components of the vertical axis (Tx, Ty) were read.

Test measurements were performed in summer. Three measurement sessions were carried out in total. Detailed information on each of the sessions are juxtaposed in Table 1.

Horizontal and vertical angles were registered during the measurements $(\mathrm{Hz}, \mathrm{V})$ and the tilt components of the vertical axis (Tx, Ty) were read from the instrument's compensation system.

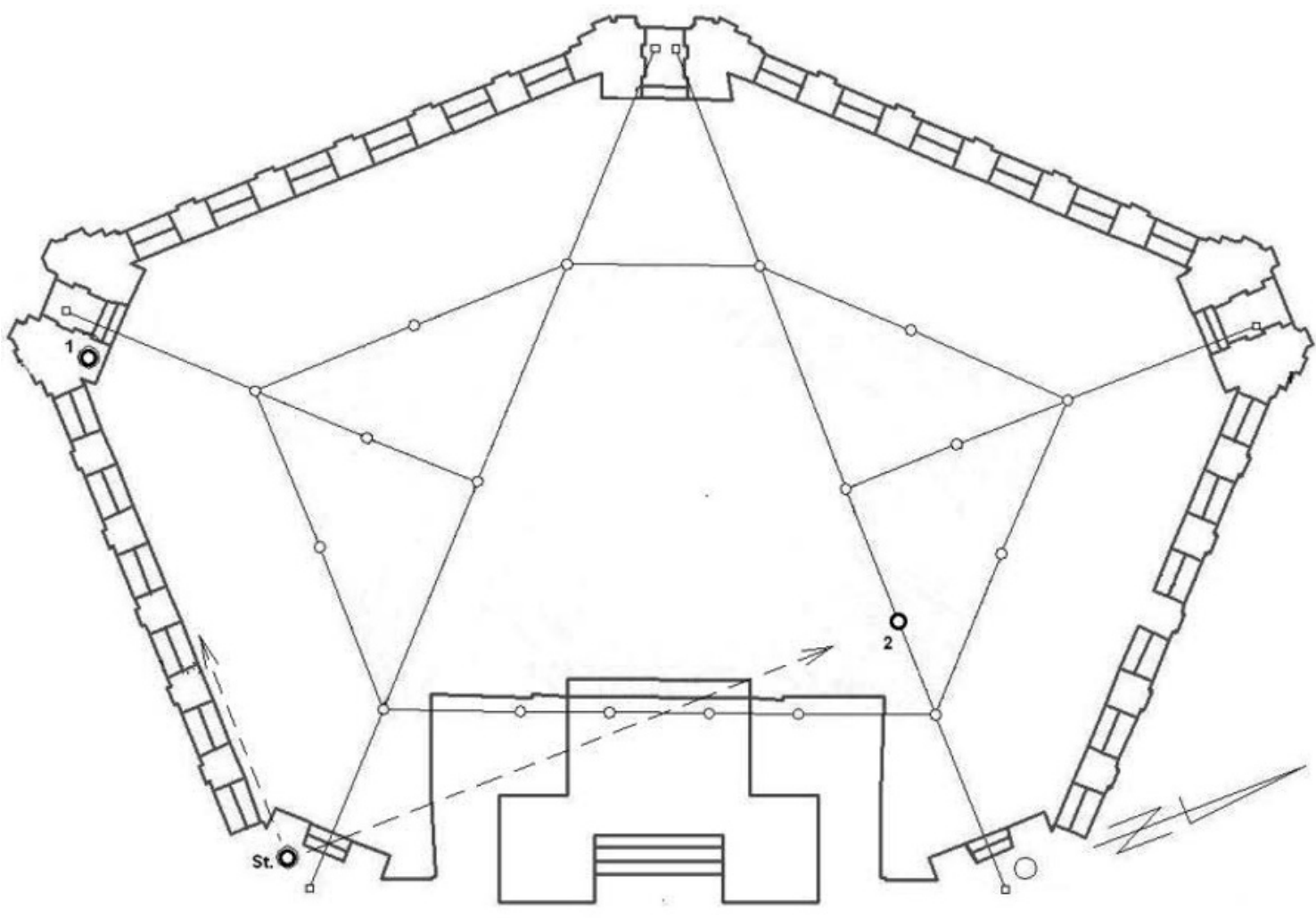

Fig. 1. Location of the station and control points during test measurements. 
Table 1. Measurement tests.

\begin{tabular}{|c|c|c|c|c|c|}
\hline No. & Type of tripod & Measurement time $[\mathbf{h}]$ & $\mathbf{T}$ min. $\left[{ }^{\circ} \mathbf{C}\right]$ & $\mathbf{T}$ max. $\left[{ }^{\circ} \mathbf{C}\right]$ & Amplitude $\mathbf{~}\left[{ }^{\circ} \mathbf{C}\right]$ \\
\hline 1 & aluminium & 64 & 20.2 & 42.8 & 22.6 \\
\hline 2 & composite & 72 & 18.4 & 40.1 & 21.7 \\
\hline 3 & wooden & 72 & 23.2 & 46.0 & 22.8 \\
\hline
\end{tabular}

\section{Processing of the measurement results}

The obtained results of the measurements were used to track the changes in the analysed parameters for the instrument and the setup. The first step of result processing involved averaging of the horizontal $\mathrm{Hz}$ and vertical $V$ angles obtained for each of the series (i).

$$
\begin{gathered}
H z_{i}=\frac{H z_{i(K L)}+H z_{i(K P)}-200^{g}}{2} \\
V_{i}=\frac{V_{i(K L)}-V_{i(K P)}+400^{g}}{2}
\end{gathered}
$$

The values for $\mathrm{Hz}$-collimation $k$ and $V$-collimation $M$ for $i$ series were determined using the following equations:

$$
\begin{gathered}
k_{i}=\frac{H z_{i(K L)}-H z_{i(K P)} \pm 200^{g}}{2} \\
M_{i}=\frac{V_{i(K L)}+V_{i(K P)}-400^{g}}{2}
\end{gathered}
$$

In each of the measurement series the values of collimation errors and zero of the vertical circle were determined twice (independently for each of the observed points).

For each point, except of horizontal and vertical angles, indications of the instrument's tilt compensation systems were registered in the form of two components: longitudinal $(T L)$ and transverse $(T C)$ referred to the instrument's system. The tilt determined in the first halfseries was noted as:

- $T L_{1 A}$ - longitudinal tilt while measuring point 1

- $T C_{1 A}-$ transverse tilt while measuring point 1

- $T L_{2 A}$ - longitudinal tilt while measuring point 2

- $T C_{2 A}-$ transverse tilt while measuring point 2

The tilt determined in the second half-series was noted analogically $T L_{1 B}, T C_{1 B}, T L_{2 B}, T C_{2 B}$.

The way of utilising measured instrument tilts to determine orientation of total station vertical axis is described in [15]. Because the directions to points 1 and 2 form a right angle (Fig. 1), the tilt components of the instrument towards each of the points can be determined 4 times. Thus, the tilt of the instrument's vertical axis towards point $1(T x)$ will be equal to:

$$
T x=T L_{1 A}=-T C_{2 A}=-T L_{1 B}=T C_{2 B}
$$

Analogically, the tilt in the direction of point 2 (Ty) will be equal to:

$$
T y=T C_{1 A}=T L_{2 A}=-T C_{1 B}=-T L_{2 B}
$$

Mean value of all four determinations will be assumed as the final value:

$$
\begin{aligned}
& T x=\frac{T L_{1 A}-T C_{2 A}-T L_{1 B}+T C_{2 B}}{4} \\
& T x=\frac{T C_{1 A}+T L_{2 A}-T C_{1 B}-T L_{2 B}}{4}
\end{aligned}
$$

It has to be noted, that the determined values of the tilts, due to the use of both the locations of the alidade, are free from the influence of the fixed error (zero point) of the compensation system.

In order to determine the zero points of the compensation system one uses the readings of the appropriate tilts (longitudinal and transverse) to the same point in different placement of the alidade (half-series).

$$
\begin{aligned}
& T L_{0}=\frac{T L_{1 A}+T L_{1 B}}{2}=\frac{T L_{2 A}+T L_{2 B}}{2} \\
& T C_{0}=\frac{T C_{1 A}+T C_{1 B}}{2}=\frac{T C_{2 A}+T C_{2 B}}{2}
\end{aligned}
$$

The final values for a measurement series took the form of a mean value of all the corresponding calculations.

$$
\begin{aligned}
& T L_{0}=\frac{T L_{1 A}+T L_{1 B}+T L_{2 A}+T L_{2 B}}{4} \\
& T C_{0}=\frac{T C_{1 A}+T C_{1 B}+T C_{2 A}+T C_{2 B}}{4}
\end{aligned}
$$

The change in the averaged horizontal angle from the measurement point to point 1 was assumed as the measure of the changes in the orientation of the tripod. The point was located on a concrete prop supporting the skylight and can be treated as fixed.

\section{Study of changes in the instrument's parameters}

As previously mentioned in chapter 1, the instrument's performance is described by four parameters. Two of them relate to the position of the line of sight (electronic) in relation to the telescope's casing. The remaining two are the zeroes of the instrument's tilt compensation system. Due to the fact that those parameters are not dependent on the used tripod, each of the measurement sessions can be used for independent determination of the listed parameters.

Because each of the sessions yielded concordant results, the author decided to present the result of a 
single measurement session no. 1 carried out using the aluminium tripod.

As it can be observed in Fig. 2 and 3, despite large temperature fluctuations the changes in the Hz- and Vcollimation are relatively small. It stands in contrast with the analogical results obtained for TDA5005 total station [9]. The course of changes in the line of sight indicates a small dependency of the changes in the measured value on the temperature. However, the changes are minimal in relation to the accuracy of the measurement, so no unequivocal conclusion can be drawn.

The analysis of the changes in the zeroes of the instrument's tilt compensation system yielded more interesting results. The graph presented in Fig. 4 shows that the instrument's tilt compensation system is characterised by different behaviour for different components. As far as the zero for the transverse component $(T C)$ is concerned, it is virtually resistant to temperature changes. However, in the case of longitudinal component $(T L)$, a clearly visible fluctuations tied to the temperature changes with the amplitude equal to approx. $13^{\text {cc }}$ can be observed. Correlation between zero position for the $T L$ component and the temperature is confirmed by Pearson correlation coefficient (Fig. 5). Minor decentration of the graph indicates that the changes in the parameters of the compensation system occur with a slight delay (approx. $2.5-3 \mathrm{~h}$ ) in relation to the temperature change.

Further research is required in order to explain the occurrence of this phenomenon.

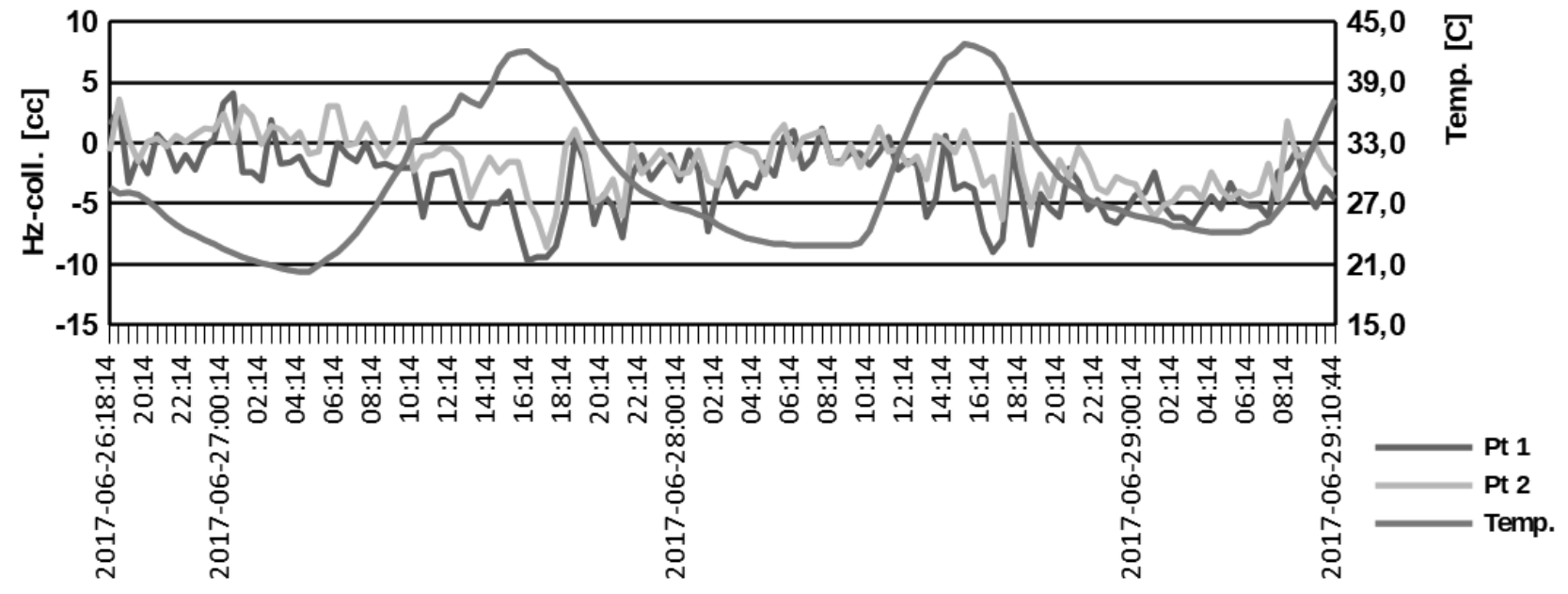

Fig. 2. Changes of Hz-collimation.

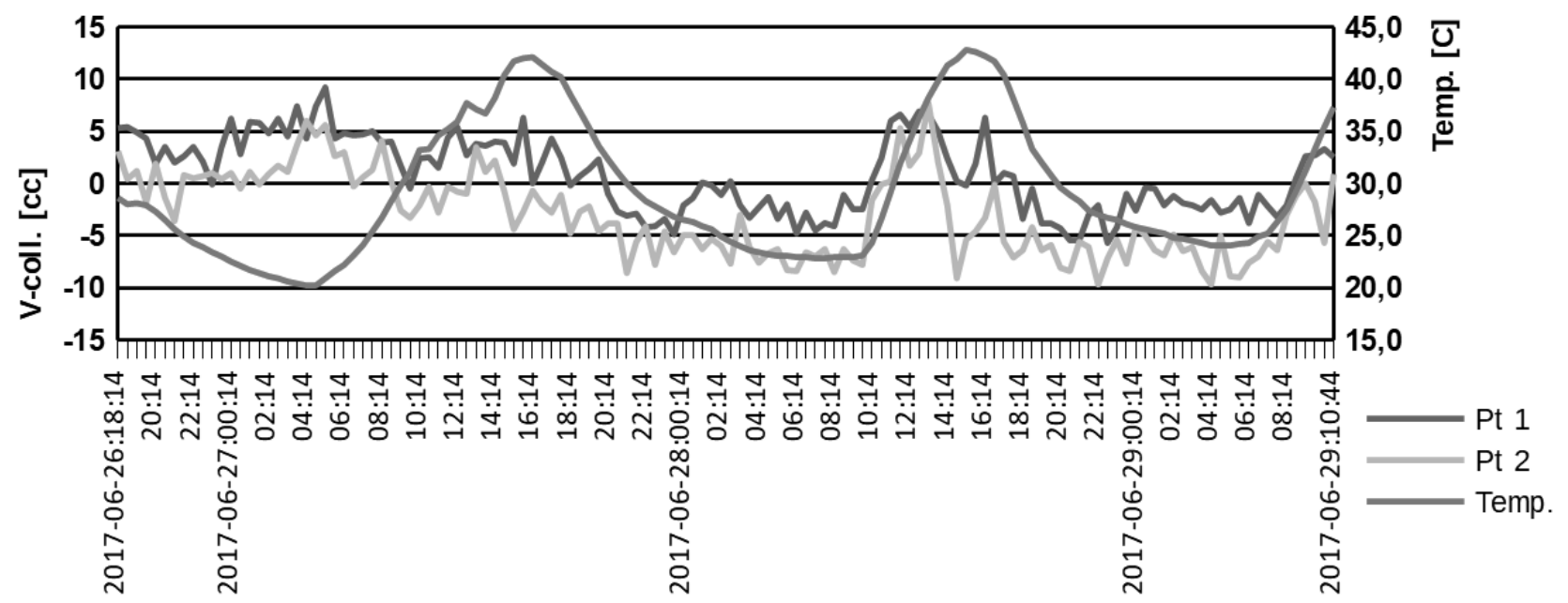

Fig. 3. Changes of V-collimation. 


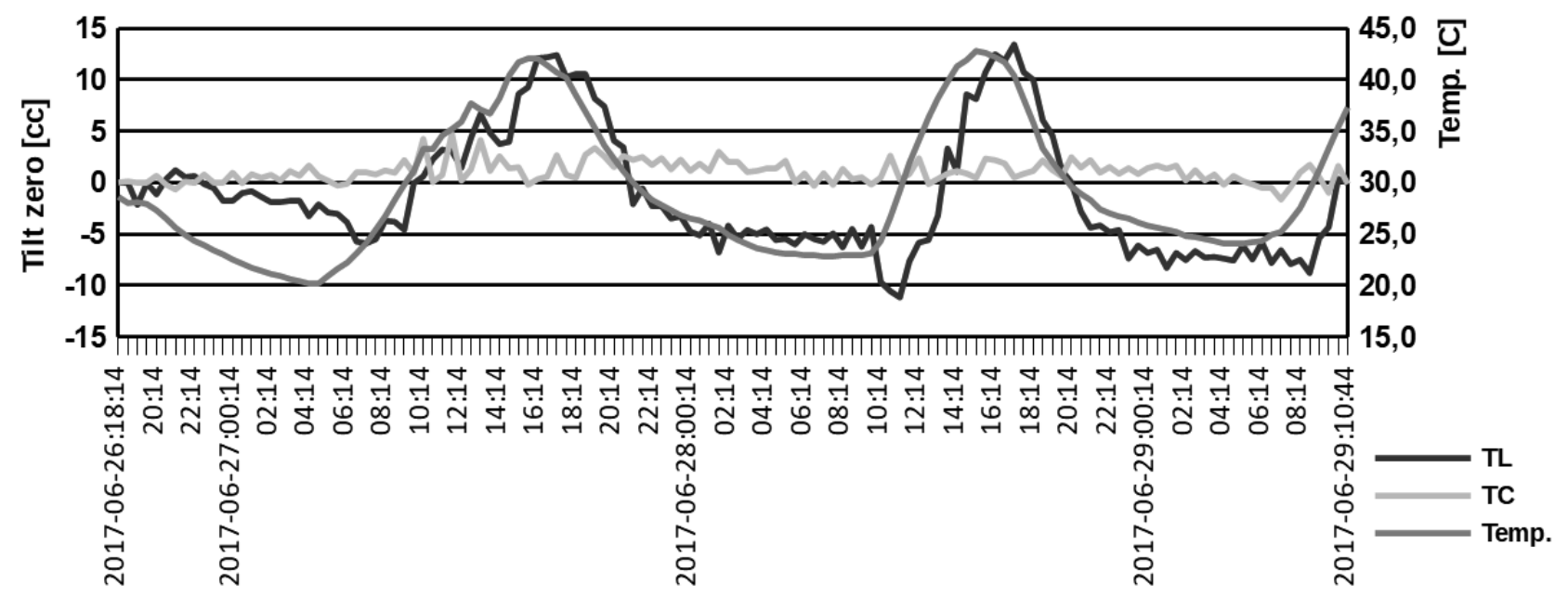

Fig. 4. Changes of tilt zero point of compensation system.

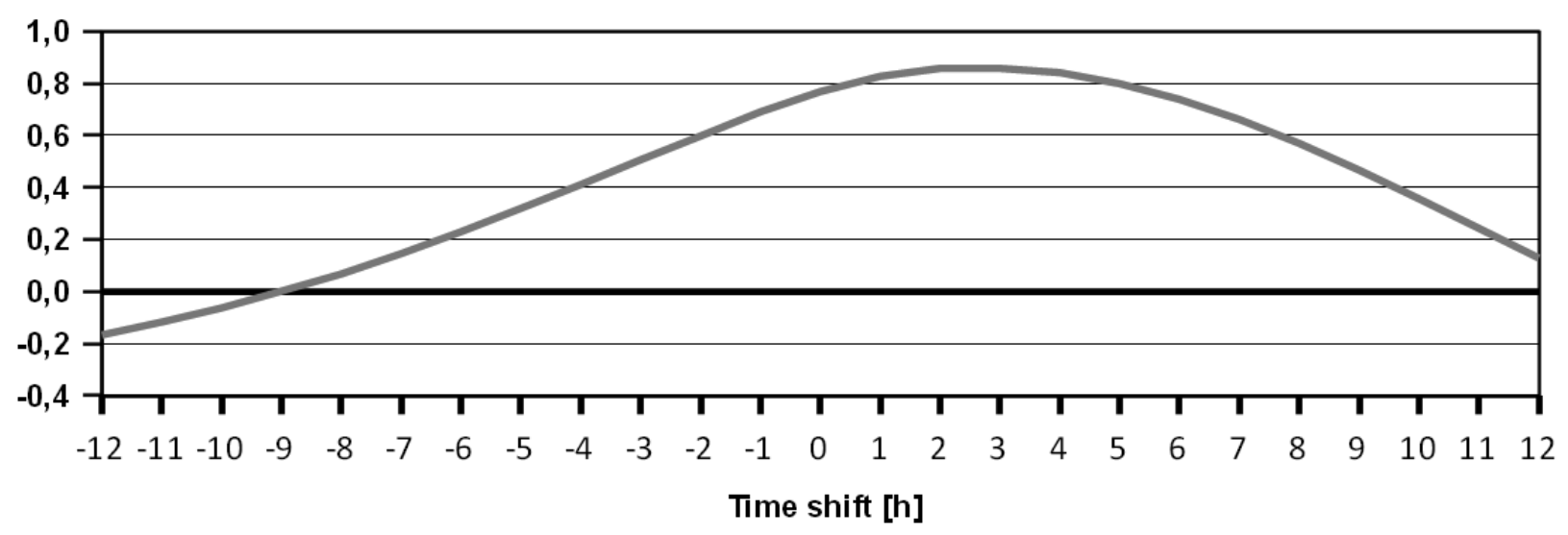

Fig. 5. Correlation between $T L_{0}$ and temperature (Pearson coefficient).

\section{Study of tripod stability}

The scope of this research also included stability checks for the selected types of tripods. Using the function of GeoCom interface of the TCRP1201+ instrument the changes in the total station's vertical axis tilt $(T x, T y)$ identified as changes in the orientation of the plane of the tripod's head were scrutinized. Change in the horizontal angle measured towards the assumed fixed point 1 was the torsion of the tripod. Changes in the tilt of the vertical axis of the instrument for each of the tripods are presented in Figs. 6, 7 and 8.

In order to facilitate the interpretation of the presented data red colour was used to track temperature fluctuations.

Figures 9, 10 and 11 present the changes in horizontal orientation for each of the successive measurement sessions. 


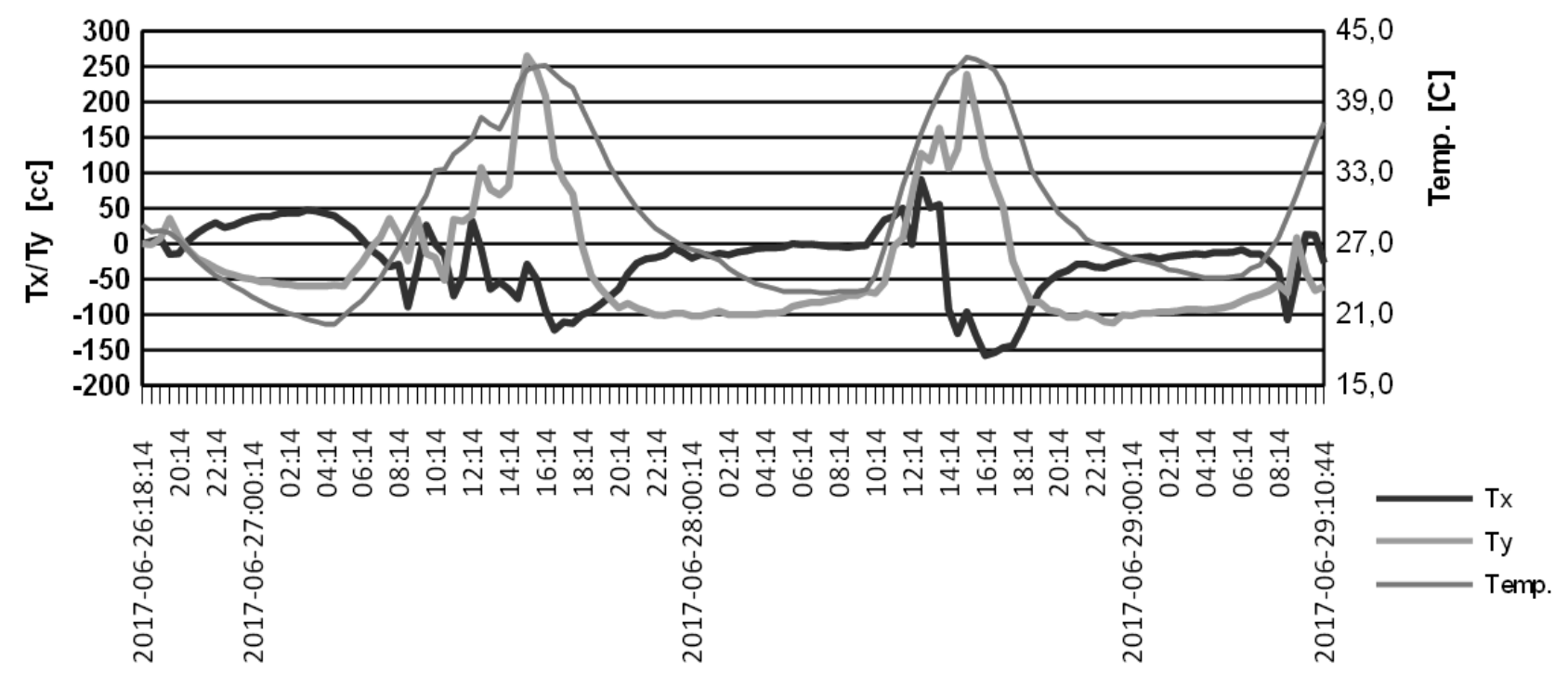

Fig. 6. Changes of instrument tilts for aluminium stand.

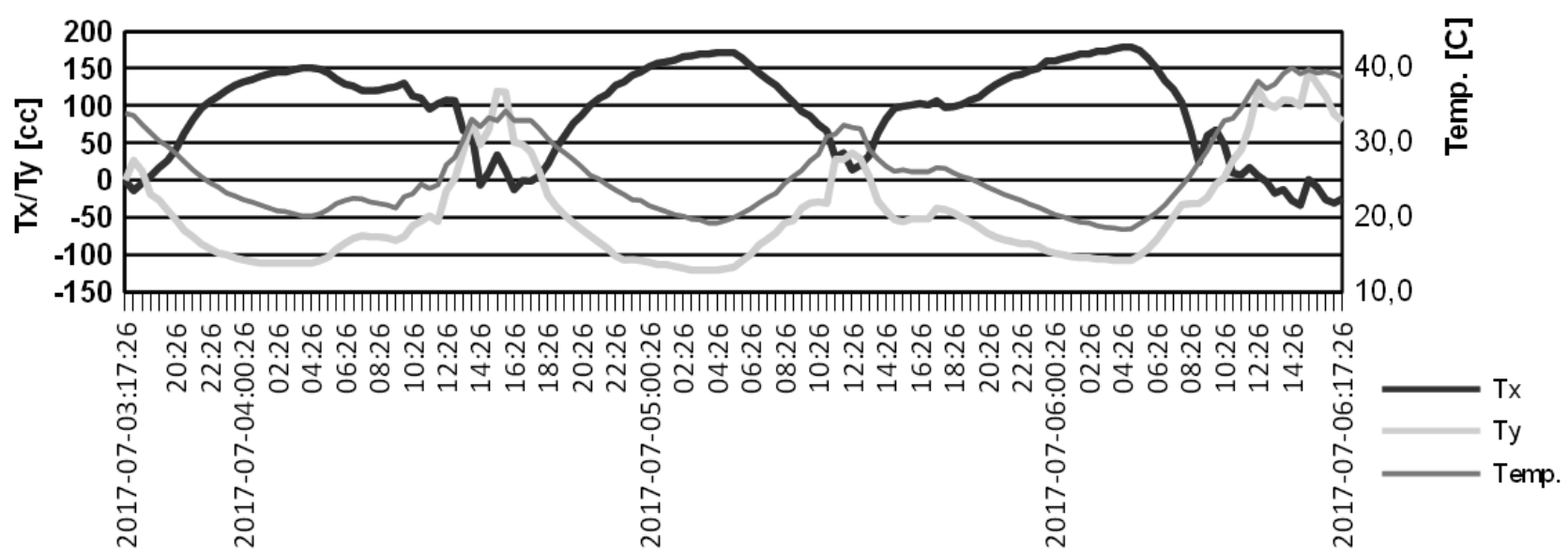

Fig. 7. Changes of instrument tilts for composite stand.

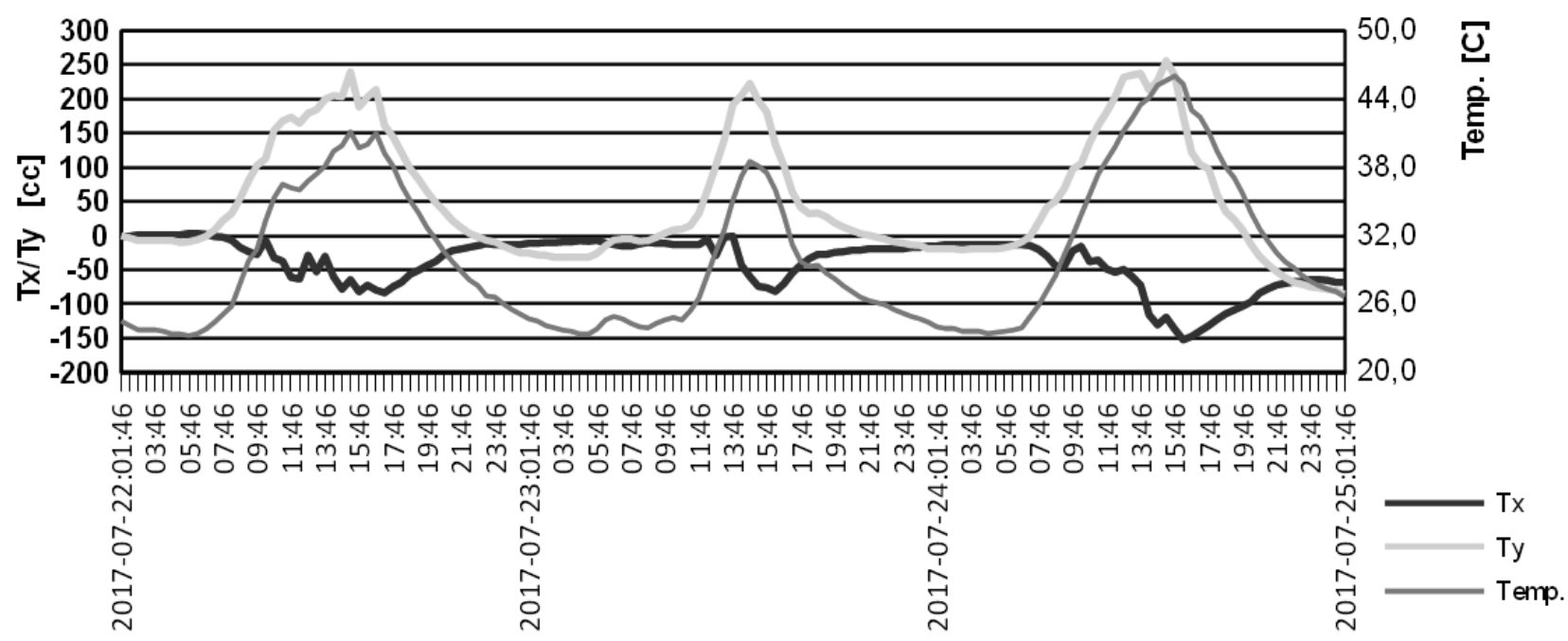

Fig. 8. Changes of instrument tilts for wooden stand. 


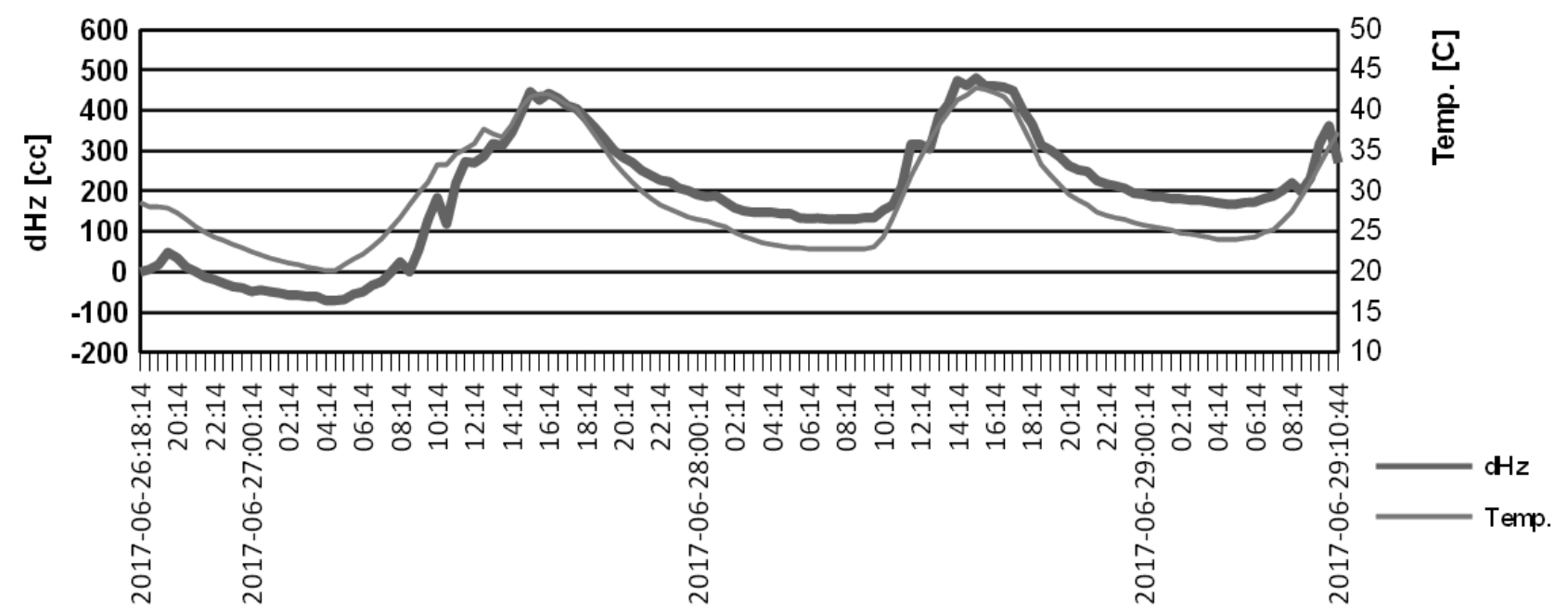

Fig. 9. Changes of instrument orientation for aluminium stand.

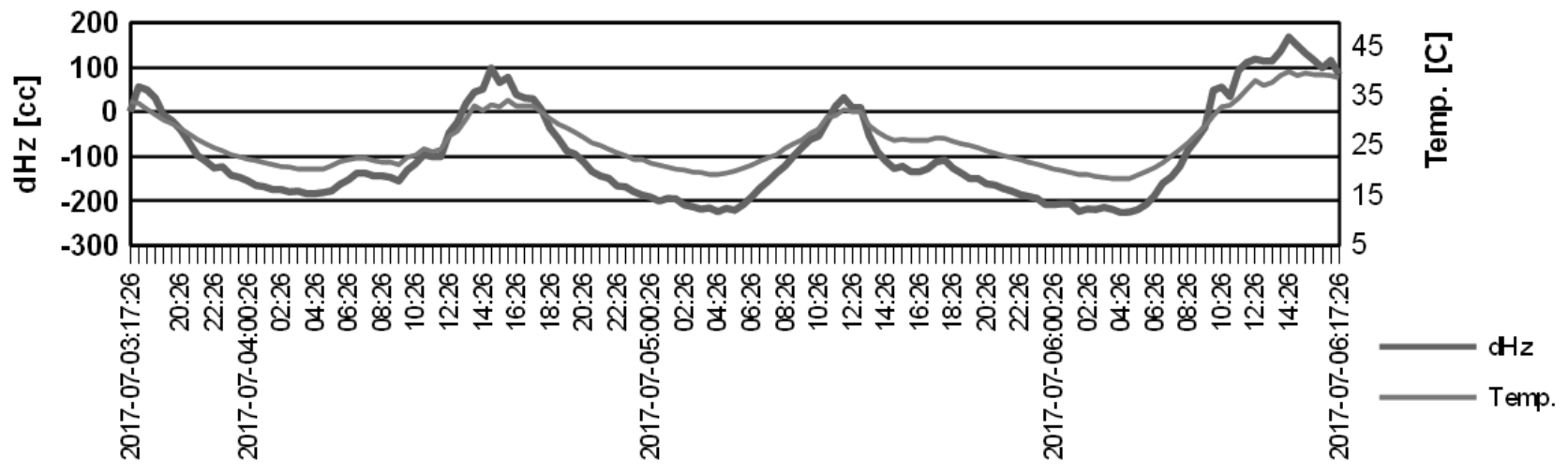

Fig. 10. Changes of instrument orientation for composite stand.

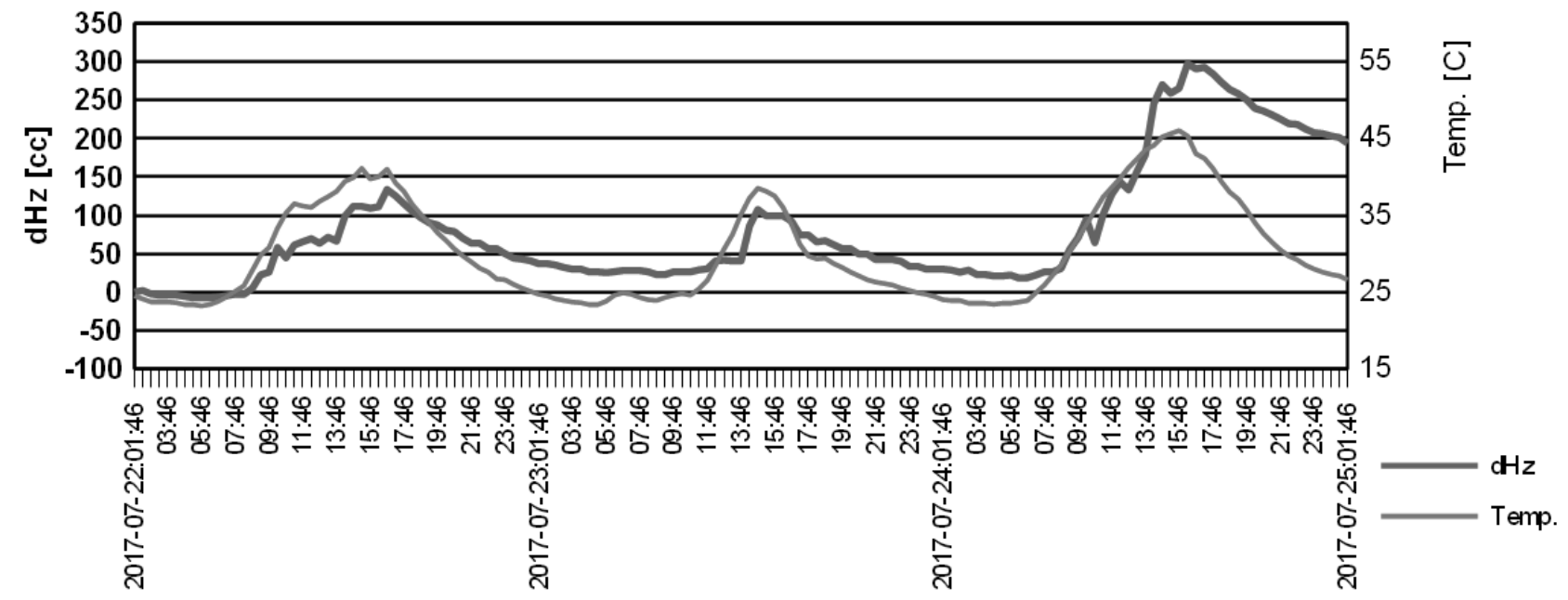

Fig. 11. Changes of instrument orientation for wooden stand.

It can be easily noticed, that both the changes in the tilt of the instrument's vertical axis are closely associated with the temperature changes. This fact is confirmed by the values of the Pearson correlation coefficient presented in Figs. 12, 13 and 14.

The tilt values are rather high, and in the case of the aluminium and wooden tripods they reached $250^{\mathrm{cc}}$.
Slightly smaller tilt values were noted for the composite tripod. Moreover, it is noticeable, that regardless of the type of the tripod the tilt signs were the same, which may be connected to the direct solar operation (the tripods were not shielded from the sunlight).

Change in the orientation for each of the tripods was also clearly connected to the temperature fluctuations, 
which was confirmed by the analyses of the correlation coefficient presented in Fig. 15. By analyzing the orientation change for each of the tripods it can be noticed that apart from repeatable changes, a certain steady, approximately uniform (Fig. 9) or variable in time (Fig. 11), trend is observable. Consequently, after the temperature returned to the initial value the instrument positioned on a tripod was orientated differently.

The carried out tests show that the smallest changes in geometry occurred for the composite tripod. Furthermore, those changes are, to the greatest extent, tied to the temperature (Figs. 13, 15). The wooden tripod retained stability for most of the time. However, during the last day of the tests in the period characterized by the highest temperatures an increased change in its geometry occurred for reasons unknown, which influenced the results of statistical analysis and lowered the Pearson correlation coefficient. Analysing Figs. 12 and 14 (aluminium and wooden tripods) it can be noticed, that $T x$ and Ty changes are similarly shifted in relation to changes of temperature. In author's opinion influence of direct sunlight on the tripods geometry can be taken into consideration as an explanation of that similarity.

When summarizing the obtained results it has to be noted, that the character of changes in tripod geometry depends not only on the type of material used for its manufacture, but also it is, to a large extent, an individual characteristic of a particular unit. Consequently, the conclusions of studies concerning tripods can be only of general nature and can only relate to orders of magnitude and the character of the said changes.

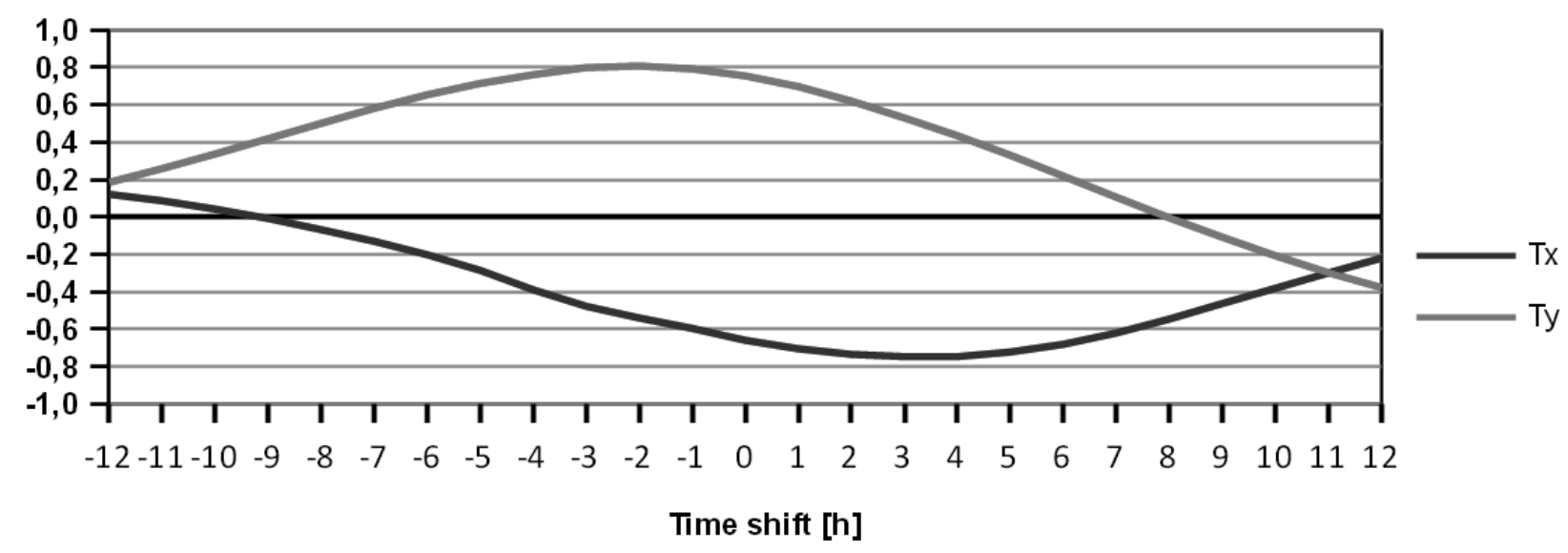

Fig. 12. Correlation between instrument tilts and temperature for aluminium stand (Pearson coefficient).

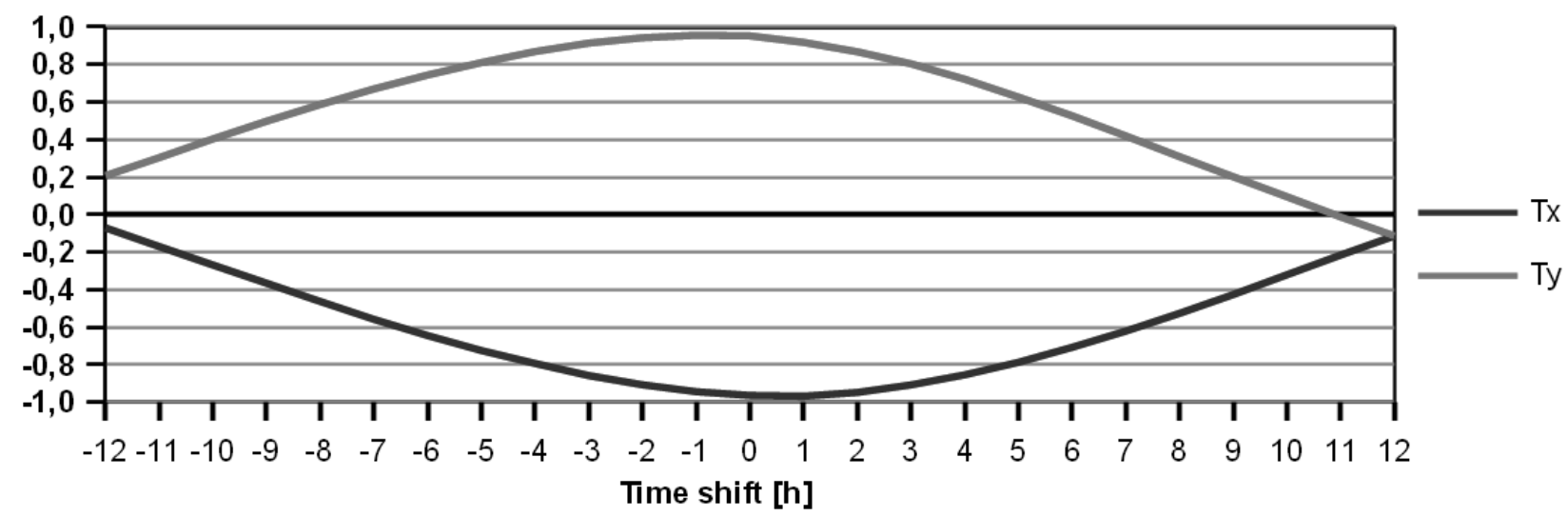

Fig. 13. Correlation between instrument tilts and temperature for composite stand (Pearson coefficient). 


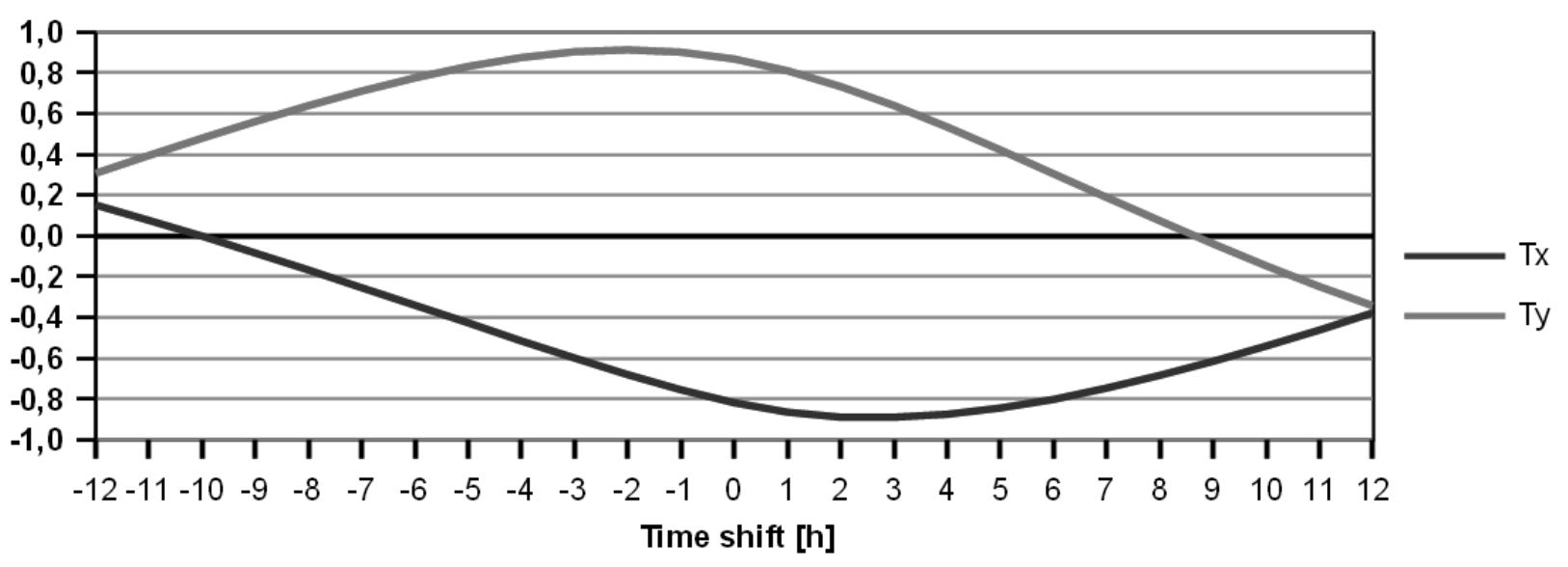

Fig. 14. Correlation between instrument tilts and temperature for wooden stand (Pearson coefficient).

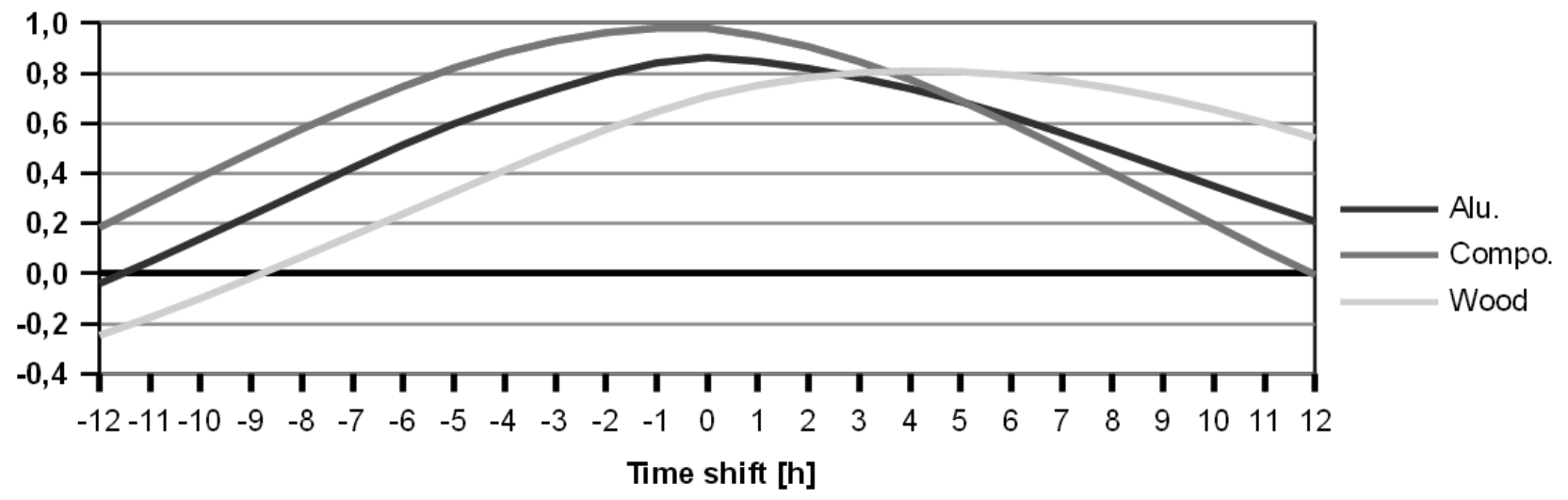

Fig. 15. Correlation between changes of orientation and temperature (Pearson coefficient).

\section{Summary}

One can draw the following conclusions on the basis of the obtained measurement results:

The studied instrument (TCRP1201+) turned out to be relatively highly resistant to fluctuations of external temperature, which makes measurements in one face a viable option. This possibility is further supported by the fact, that in the real field conditions the temperature fluctuations will rarely reach such high values as those observed during the test measurements. It should be noted however, that the one face measurement should be treated as the last resort and be used only in situations where there is no possibility to carry out full measurement series.

The measurement in two faces is also supported by the results of study on the behaviour of the total station's vertical axis tilt compensation system. As proven by the tests, the system is partially (one component) susceptible to temperature fluctuations. Measurement in two faces makes it possible to eliminate errors caused by the tilt compensation system.

The study on tripod stability revealed that the changes in the geometry of standard tripods used in geodetic surveys are significant and in the case of an extended measurement have to be taken into consideration.
The performed measurement tests were accompanied by large temperature fluctuations. That translated into significant geometry value changes in the inspected tripods, which was confirmed by the analyses of Pearson's correlation coefficient. The composite tripod turned out to be the most stable among all the tested variants. It was the result of moderate geometry value changes as well as their highest correlation with temperature fluctuations. It does not, however, change the conclusion, that in the case of all of the tripods those changes were significant in relation to the angle measurement accuracy required in the control measurements of engineering structures.

Although the changes in the tilt of the tripod's head translating into changes in the tilt of the instrument's vertical axis usually do not exceed the range of its compensation system, the torsion of the tripod resulting in the change of the horizontal orientation ought to be taken into account during the measurement design stage. Periodic measurements towards fixed point placed outside the object's zone of influence can be used for that purpose.

Regardless of the above, in the case of long-term measurements one should aim to use heavy tripods with reinforced construction which guarantee higher level of stability for the instruments setup. 


\section{References}

1. V. Michel, T. Person, M. Kasser, Surveying in Industry and Construction FIG Working Week, TS22.1 (2003)

2. A. Berberan, M. Machado, S. Batista, Surv. Rev., 39(305), 203-211 (2007)

3. W. Stempfhuber, Bautechnik, 89(11), 794-800 (2012)

4. T. Owerko, M. Strach, Rep. on Geod., 87(2)/2009, 291-299 (2009)

5. D. S. Paraforos, M. Reutemann, G. Sharipov, R. Werner, H. Griepentrog, Computers and Electronics in Agroculture 136/2017, 166-175 (2017)

6. J. Vaněček, M. Štroner, Acta Polytechnica 56(6), 478-491 (2016)

7. M.Woźniak, W. Odziemczyk, Rep. on Geod. and Geoinf., 104/2017, 79-90 (2017)

8. M. Woźniak, W. Odziemczyk, S. Jastrzębski, J. Wojciechowski, Rep. on Geod., 1(84), 105-110 (2008)

9. M. Woźniak, W. Odziemczyk, Rep. on Geod. 1(85), 105-110 (2009)

10. W. Odziemczyk, Rep. on Geod. and Geoinf., 97/2014, 35-46 (2014)

11. H. Kirschner, W. Stempfhuber, (Proceedings of the 1st International Conference on Machine Control \& Guidance, 51-60, 2008)

12. M. Woźniak, W. Odziemczyk, Wykorzystanie metod geodezyjnych $w$ ocenie stanu geometrycznego budowli (Publishing House of Silesian University of Technology, 173-180, 2008, in Polish)

13. W. Odziemczyk, Inżynieryjne Zastosowania Geodezji (Publishing House of Poznan University of Technology, 2012, in Polish)

14. C. Depenthal, Allgem. Verm.-nachrichten 6/2004, 227-233 (2004)

15. P. Raby, U.S. Patent No. 6,243,658 (2001) 